\title{
Brain Tumor Segmentation using Watershed Technique and Self Organizing Maps
}

\author{
Ashima Anand* \\ Department of Electronics and Communication, Chandigarh University, Mohali - 140413, Punjab, India; \\ ashima.anand327@gmail.com
}

\begin{abstract}
Objectives: To segment tumor with higher accuracy. Methods/Statistical Analysis: Noise removal is done with the help of Gabor filter as a preprocessing step. Skull stripping is done to remove non cerebral regions using thresholding and morphological operations. Segmentation using watershed algorithm is done, as it achieves exact location of outline. Unsupervised type of neural network i.e. self organizing maps is used for classification. Finding: It has been analyzed that by combining watershed and neural networks segmentation accuracy has been improved to $95.93 \%$. The motive of the research is to segment the tumor with precision using computerized segmentation algorithm that can help physicians to analyze brain diseases and treatment can be started as soon as possible. Applications: The proposed technique can be used in image processing of brain tumor detection.
\end{abstract}

Keywords: Brain Tumor Segmentation, Image Segmentation, Magnetic Resonance Imaging (MRI), Self Organizing Maps (SOM), Stationary Wavelet Transform (SWT)

\section{Introduction}

Tumors may be grouped into primary and secondary ${ }^{1}$. Primary or brain metastasis tumors may initiate in the brain or membranes, nerves or glands which is further categorized into Benign (not causes cancer) and Malignant (prone to cancer). Malignant brain tumor is characterized as threatful, which invade rapidly, destroying brain cells by causing swelling. The exact cause of brain tumors is not clear.

Brain tumor is detected by medical examination through various imaging modalities such as CAT and $\mathrm{MRI}^{2}$. Segmentation involves the process of splitting up the image into distinct regions i.e. according to criteria of homogeneity ${ }^{3}$. Segmentation of brain tumor is one of the competitive tasks since tumor's characteristics are very difficult to visualize ${ }^{4}$. Various challenges related to tumor segmentation are high diversity appearance and inconsistent shape. Segmentation of tumor done in a manual manner by doctors is a weary task which shows variations when diverse doctors undergo the same task of segmentation.
The motive of the research is to segment the tumor with precision using computerized segmentation algorithm that can help physicians to analyze brain diseases.

$\mathrm{In}^{5}$ provides a model for segmentation of tumor pictures. Along with detection of tumor, edema is additionally detected at an equivalent time. In ${ }^{\underline{6}}$ authors performed brain tumor segmentation based on coefficient known as Apparent Diffusion Coefficient (ADC).Combined methods of unsupervised type Artificial Neural Networks (ANN) and wavelet is being used. $\operatorname{In}^{7}$ authors discussed about automatic segmentation of non-homogeneous image data and focus on filling up gap between bottomup and top-down generative approach. This paper focus on formulation using Bayesian model for building of model into evaluation of affinities. In ${ }^{\underline{8}}$ authors planned an automatic tumor segmentation technique supported Convolution Neural Networks (CNNs). In 9 authors proposed a method based on intelligent Neural Networks $(\mathrm{NN})$ which classifies numerous brain tumors varieties.

${ }^{*}$ Author for correspondence 
The layout of this paper is as follows: Section 2 relates with material and methods. Section 3 explains results and discussions. Section 4 concludes the paper.

\section{Materials and Methods}

Proposed Flow Chart is shown in Figure 1. The implementation steps of algorithm are as follows:

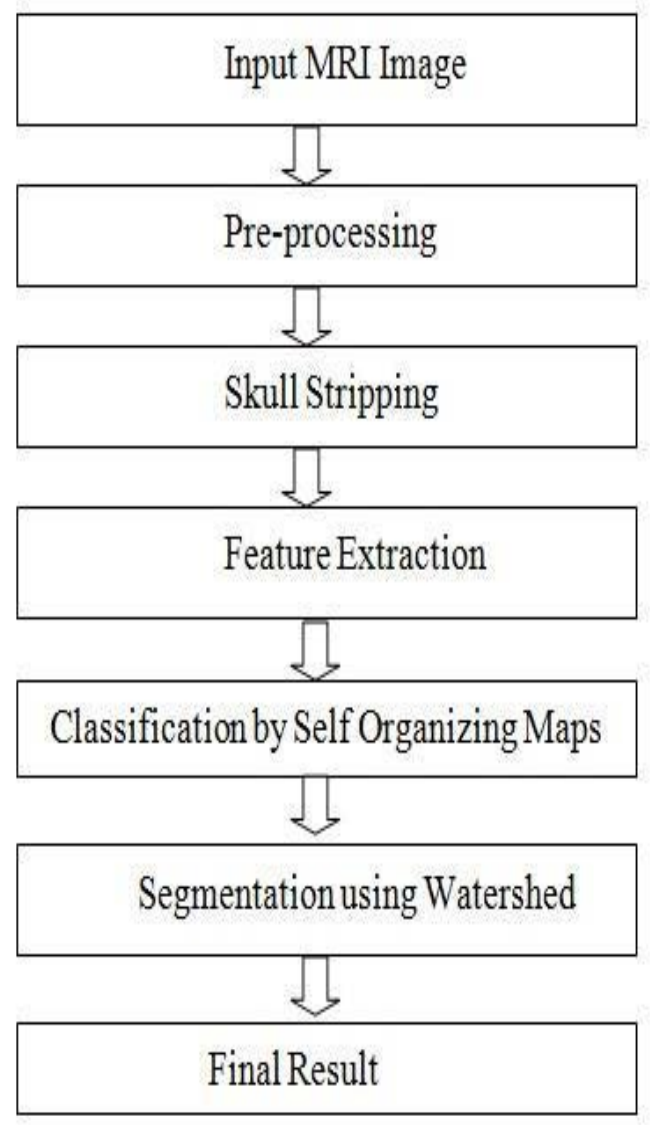

Figure 1. Flowchart of proposed methodology.

\subsection{Preprocessing}

It refers to the reduction in data set and used for optimization of varied parameters ${ }^{10}$. It is basically a pre-processing step to remove noise in the input image. Gabor filter is used in the preprocessing stage. This filter is used for edge detection and is similar to human visual system ${ }^{\underline{11}}$.

\subsection{Skull Stripping}

Skull stripping is executed before pre-processing, which eliminates non-cerebral regions inside the brain as it is not area of our concern ${ }^{12}$. By using combined methods of thresholding and morphological operations, skull stripping is performed.

\subsection{Feature Extraction}

After skull stripping, features are extracted with the help of Stationary Wavelet Transform (SWT). SWT possess advantage over traditional wavelet transform since its coefficients will not change even if the signal is varied. Various parameters which are evaluated in the step of feature extraction are energy, entropy, standard deviation and Mean Absolute Difference (MAD). Entropy parameter represents randomness, whereas energy differentiates whether texture is broad or fine. Standard deviation depicts the mean contrast whereas mean absolute difference is basically measure of energy ${ }^{12}$.

\subsection{Training using the Self Organizing Maps (SOM)}

It is among the foremost neural network models. It supports competitive learning networks. No human involvement is needed throughout; therefore it is termed as unattended sort oflearning. Map units perform agglomeration of knowledge. It conjointly evaluates the memberships of sophistication of input file and helps in detecting features ${ }^{13}$. The SOM creates discrete mapping of input space, $Y \in S n$ uses a set of neurons. Initialization of all the weights $\{\mathrm{w} 1, \mathrm{w} 2, \mathrm{wN}\}$ is done at the beginning to small random numbers. wj is the weight vector corresponding to neuron $\mathrm{j}$ and $\mathrm{N}$ is the total number of neurons. The algorithm repeats the steps shown in Algorithm 1, where $\mathrm{n}(u, v, t)$ is the neighborhoodfunction's is the set of neuron indexes. The coefficients $\{\alpha(t), t \geq 0\}$ known as the adaptation gain decreases monotonically and satisfies the following property ${ }^{14}$.

$$
\lim _{\tau=\infty} \sum \alpha(t) \rightarrow \infty
$$

Algorithm 1 SOM Metaheuristic ${ }^{14}$.

\section{Repeat:}

1. First step is to take an input $\mathbf{y}(t)$ at time $t$ and choose the winner:

$u(t)=\operatorname{argmin} n, y(\mathrm{t})-\mathrm{wv}(\mathrm{t}) \operatorname{n},(2)$

2. Weights of the corresponding winner are updated again until convergence of map takes place:

$$
\operatorname{wv}(t)=\alpha(t) \eta(u, v, t)[\mathrm{y}(t)-\mathrm{w} u(t)](3)
$$




\subsection{Segmentation using Watershed Technique}

As shown in Figure 2, Segmentation is basically gradient based and is mostly used technique referred to as watershed algorithmic rule. Within the landscape the mountains are just like the ridgelines (i.e. high intensity) and valleys are the structure basins (i.e. Low intensity) ${ }^{15}$. This algorithmic rule leads to complete contour of pictures. It is best technique that depends on edges instead of color. Regardless of its benefits expertise over segmentation, therefore various pre or post process strategies are developed for higher segmentation results.

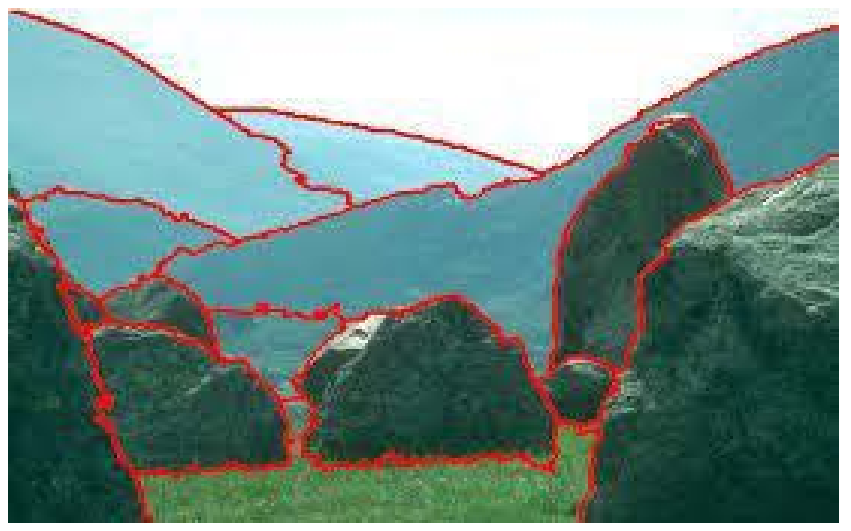

Figure 2. Segmentation using watershed algorithm $\mathrm{m}^{16}$.

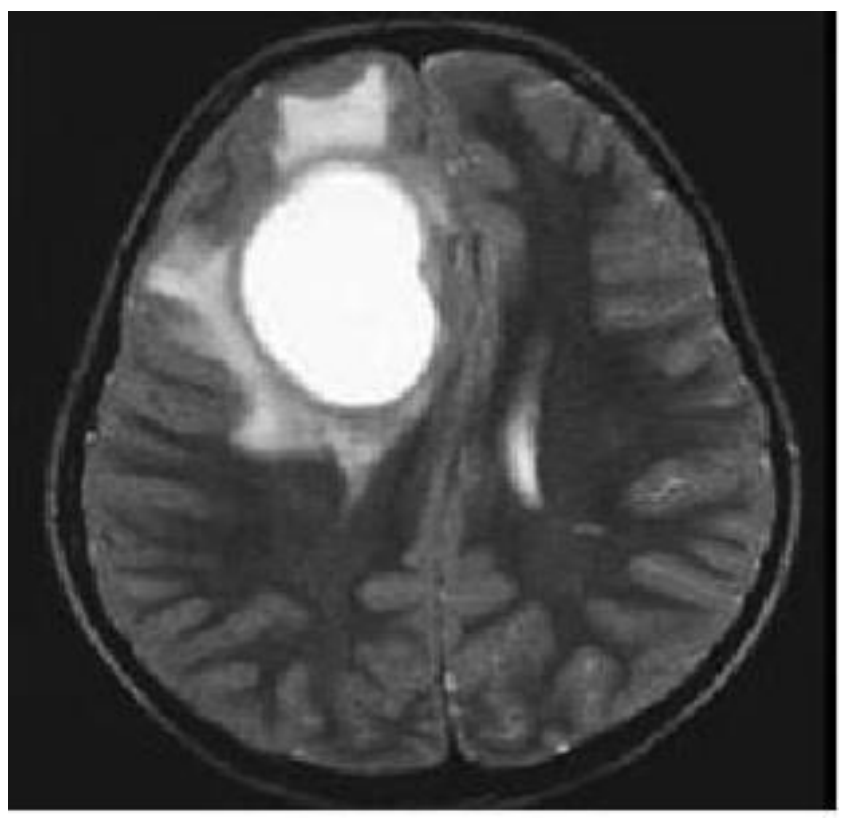

Figure 3. Input MRI image.

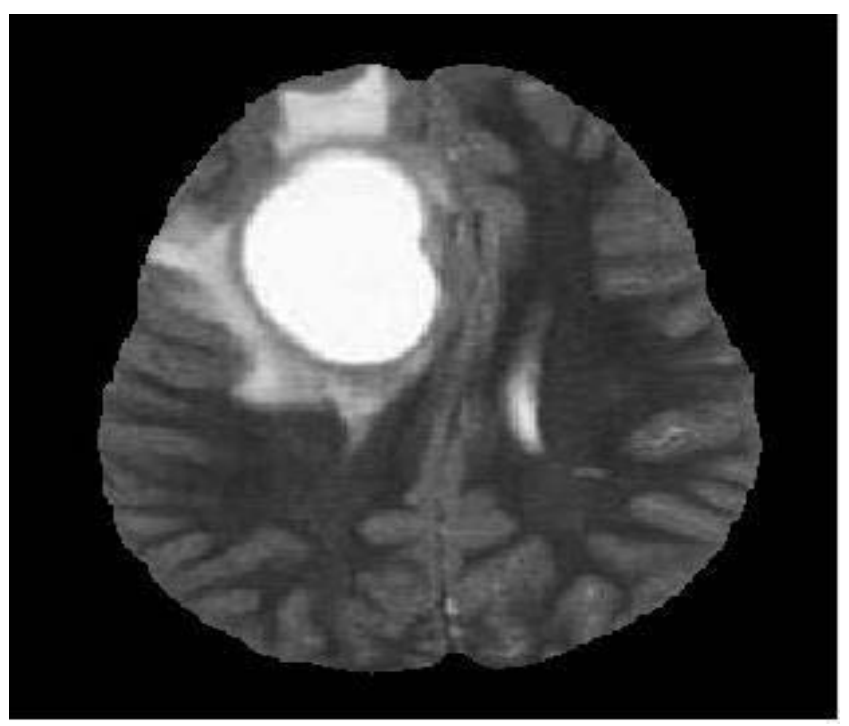

Figure 4. Image after skull stripping.

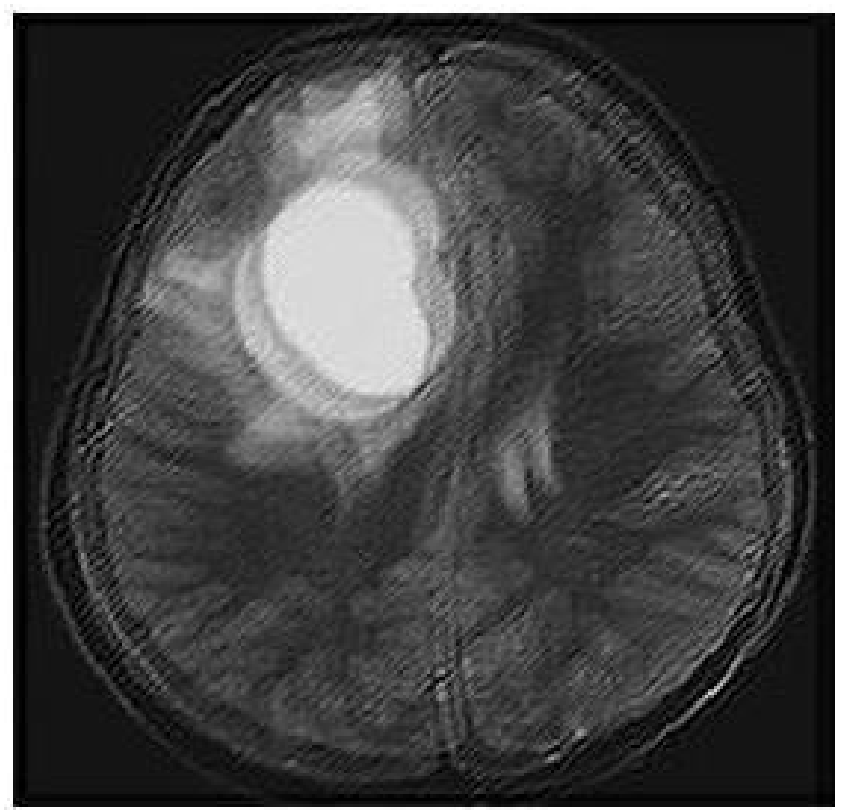

Figure 5. Gabor filtered image.

\section{Results and Discussions}

Results are evaluated on medical MRI brain image which consist of tumor. The input image is $2 \mathrm{D}$ MRI image which is basically a grey scale image as you can see in Figure 3. This is input image on which we implemented our proposed algorithm to test the tumor. Figure 4 is skull stripped image which is obtained after skull stripping. 
This step of skull stripping is executed before pre-processing, which eliminates non-cerebral regions inside the brain as it is not area of our concern. Figure 5 shows the filtered image after passing through Gabor filter.

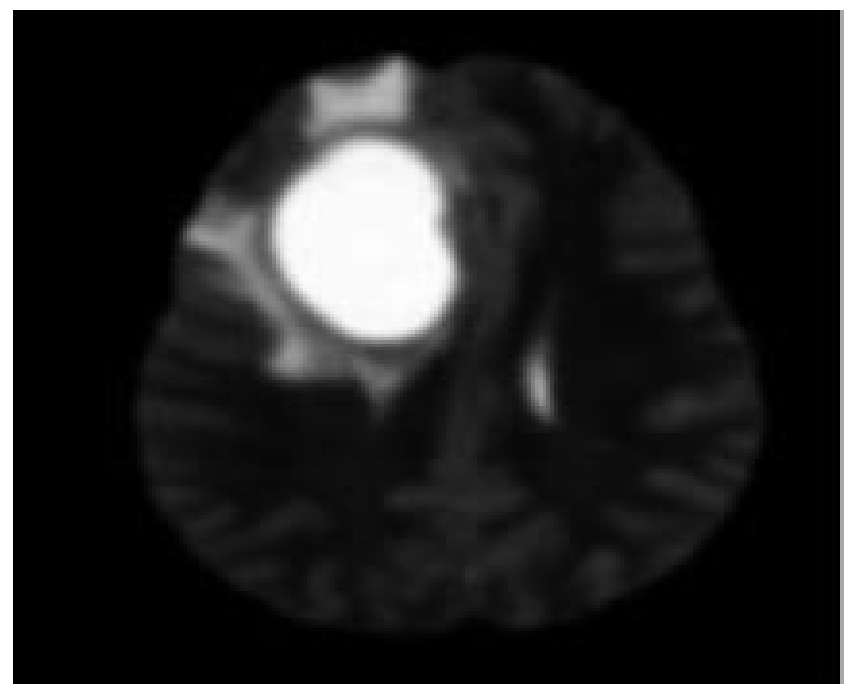

Figure 6. Extraction of energy.

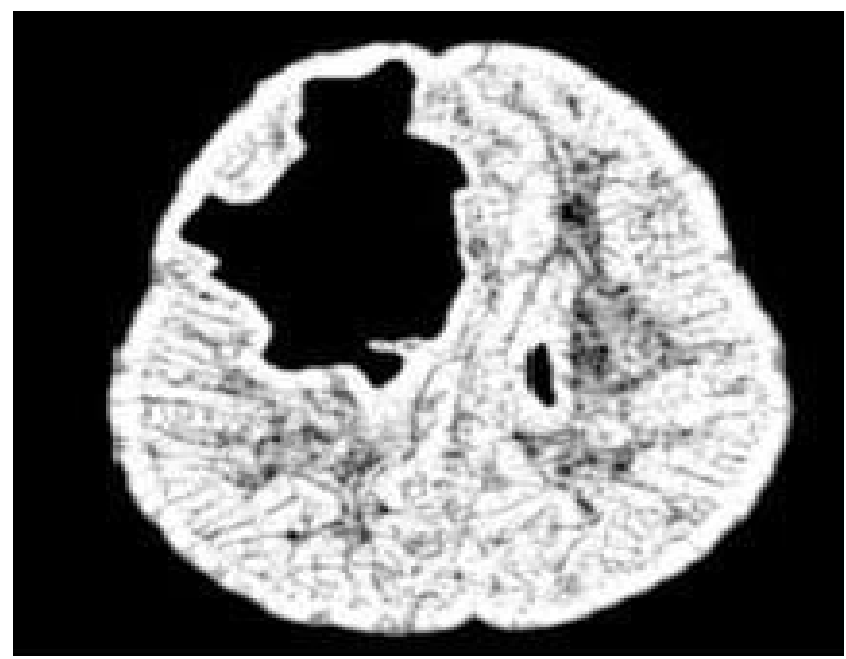

Figure 7. Extraction of entropy.

Various parameters are calculated with the help of Stationary Wavelet Transform (SWT).Figure 6 shows energy parameter in input image. Figure 7 depicts entropy where in figure white region shows larger variations. Figure 8 calculates Mean Absolute Difference which is also referred to as mean and expected value. Figure 9 shows the standard deviation. After feature extraction, features are given as input to the neural networks. These features are trained with the help of unsupervised type of neural network (i.e. Self Organizing Maps). Map forms labels in correspondence to the intensity values of the original image. Figure 10 shows the SOM labels created and Figure 11 shows the image after applying the SOM algorithm. Our focus is to segment the tumor accurately, which we achieved with the help of watershed segmentation as shown in Figure 12 and Figure 13.

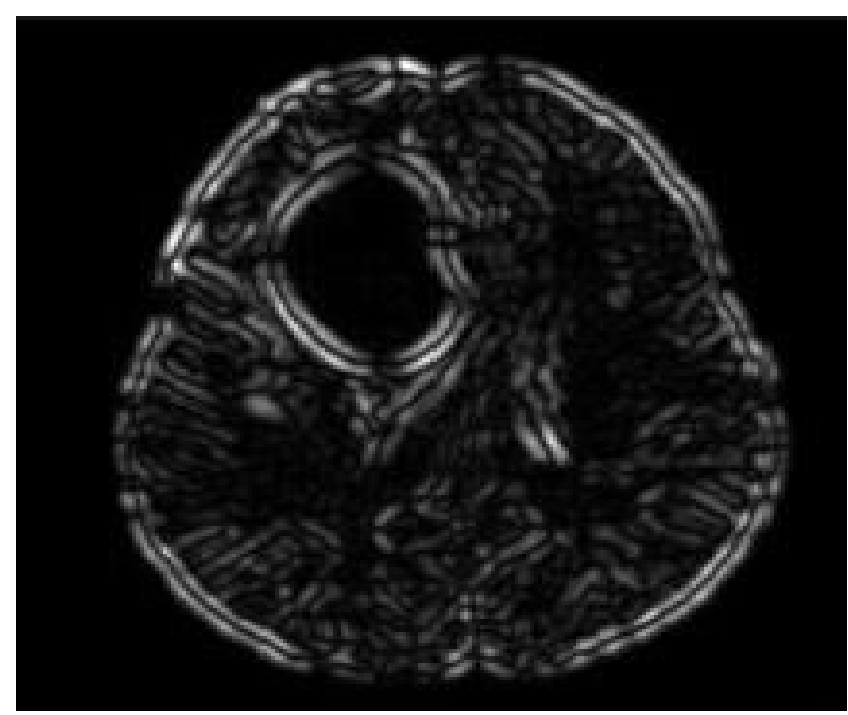

Figure 8. Mean absolute difference.

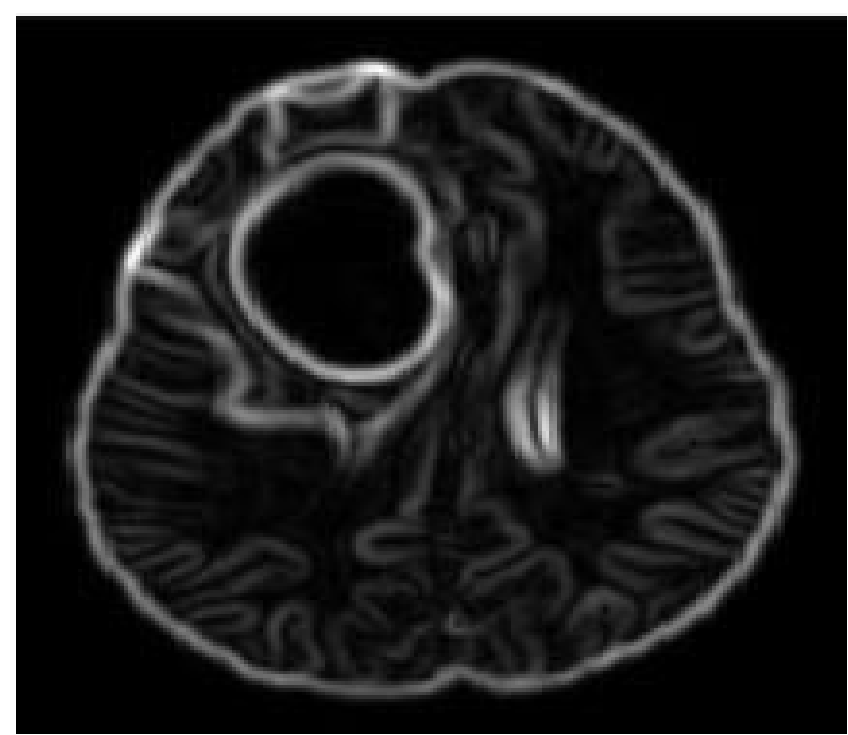

Figure 9. Standard deviation. 


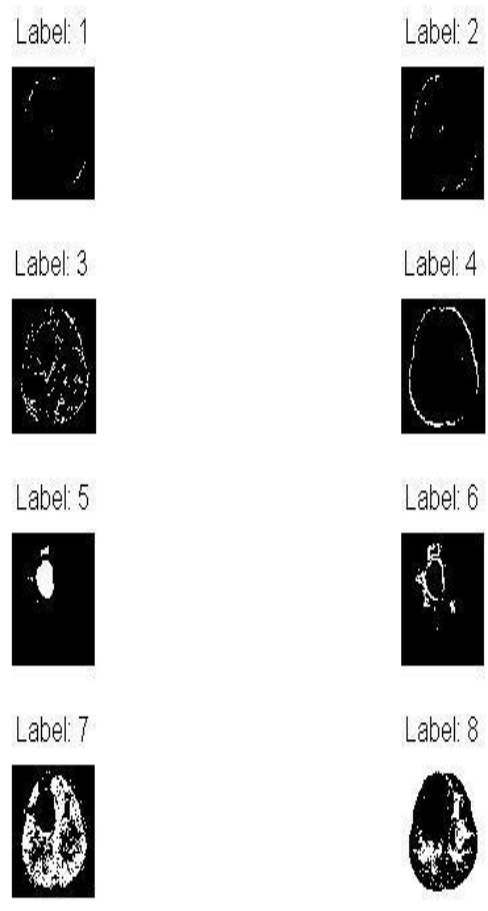

Figure10. SOM labels.

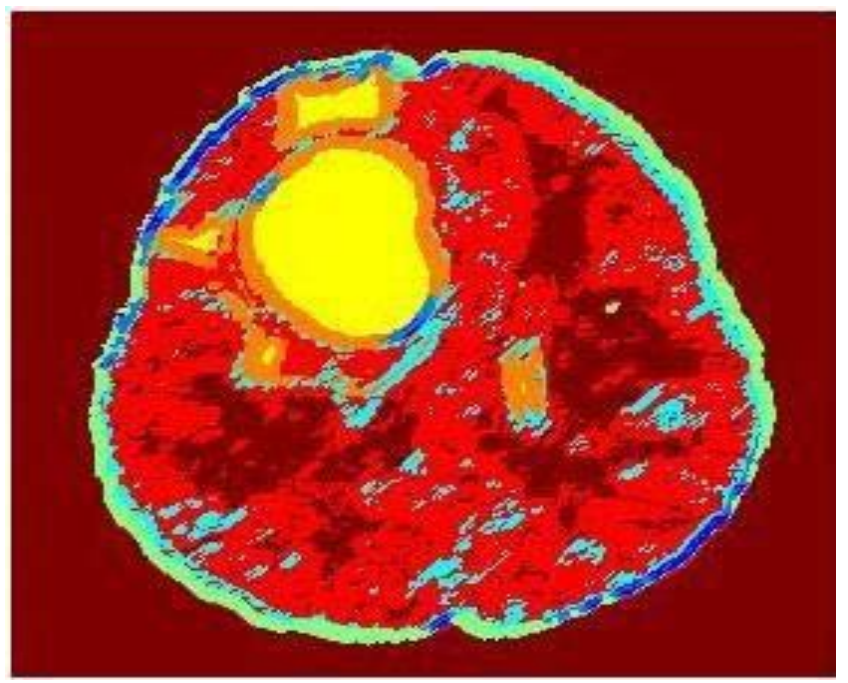

Figure 11. Image after applying SOM.
The potency of the proposed method can be elucidating by the various quantitative assessment. Assume True Positive be true positive, true negative be true negative, False Negative be false negative and false Positive be false positive. Values of True Positive, True Negative, False Positive and False Negative are shown in Table 1. Comparison of distinct performance parameters is shown in Table 2.

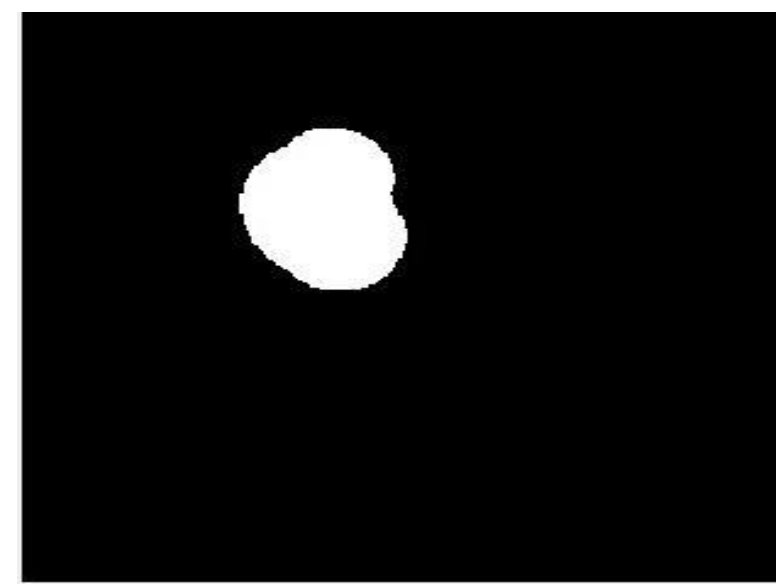

Figure 12. Tumor extraction using watershed segmentation.
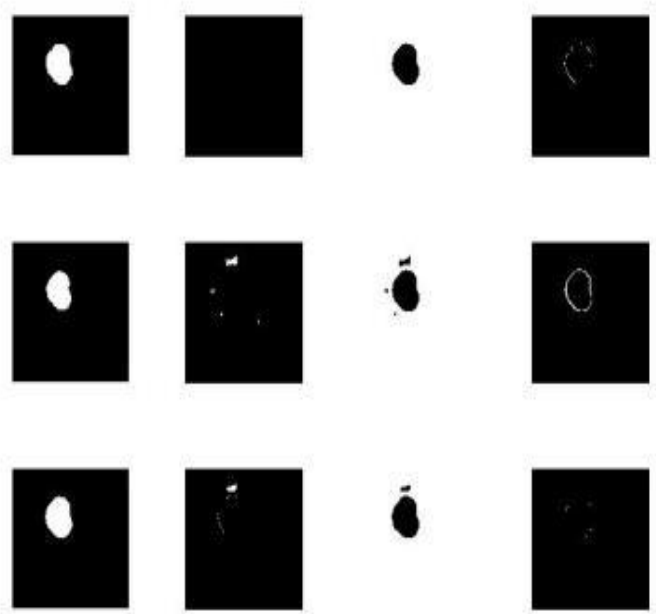

Figure 13. True positive, false positive, true negative and false negative values obtained using SOM, watershed and our technique

Table 1. Values of true positive, true negative, false positive and false negative

\begin{tabular}{|l|l|l|l|l|}
\hline & $\begin{array}{l}\text { True Positive } \\
(\mathrm{TP})\end{array}$ & $\begin{array}{l}\text { True Negative } \\
(\mathrm{TN})\end{array}$ & $\begin{array}{l}\text { False Positive } \\
(\mathrm{FP})\end{array}$ & $\begin{array}{l}\text { False Negative } \\
(\mathrm{FN})\end{array}$ \\
\hline SOM & 2964 & 61705 & 360 & 507 \\
\hline Watershed & 3421 & 61806 & 259 & 50 \\
\hline SOM + Watershed (Our Method) & 3330 & 62065 & 0 & 141 \\
\hline
\end{tabular}


Table 2. Comparison of distinct performance parameters

\begin{tabular}{|l|l|l|l|}
\hline & SOM & Watershed & SOM+ Watershed \\
\hline Accuracy (\%) & 75.02 & 91.09 & 95.93 \\
\hline Dice coefficient & 0.87 & 0.95 & 0.97 \\
\hline Sensitivity & 0.85 & 0.98 & 0.95 \\
\hline Specificity & 0.99 & 0.99 & 1 \\
\hline
\end{tabular}

Table 1 Values of True Positive, True Negative, False Positive and False Negative.

$$
\text { Accuracy }=\frac{\mathrm{TP}+\mathrm{TN}}{(\mathrm{TP}+\mathrm{TN}+\mathrm{FN}+\mathrm{FP})}
$$

Dice Coefficient $=\frac{(2 * \mathrm{TP})}{(2 * \mathrm{TP}+\mathrm{FN}+\mathrm{FP})}$

$$
\text { Sensitivity }=\frac{\mathrm{TP}}{\mathrm{TP}+\mathrm{FN}}
$$

$$
\text { Specificity }=\frac{\mathrm{TN}}{\mathrm{TN}+\mathrm{FN}}
$$

\section{Conclusion}

Segmentation of brain tumor is proposed using combined techniques of watershed and neural networks. Unsupervised type of neural networks i.e. Self Organizing Maps is used for clustering of data. Watershed algorithm is proposed since it depends on edges rather than color. Various textural parameters are calculated. The decision regarding image segmentation quality is generally subjective.

Segmentation of brain tumor using two dimensional (2D) Magnetic Resonance Images (MRI) is done. It may be worthwhile that work can be extended to three dimensional (3D) images for segmentation.

\section{Acknowledgment}

The authors express their deep sense of gratitude to Chandigarh University for conferring valuable, skilful guidance and encouragement.

\section{References}

1. Maru D, Shah B. Image segmentation techniques and genetic algorithm, International Journal of Advanced Research in Computer Engineering and Technology. 2013; 2:1483-84
2. Min L, Wang J, Wu F, Liu T, Pan Y. A survey of MRI-based brain tumor segmentation methods, Tsinghua Science and Technology. 2014; 19:578-95. Crossref

3. Gonzalez RC, Woods R. Digital image processing, 2nd Edition, 2nd Ed., Publishing House of Electronics Industry, Beijing; 2007. p. 711-12.

4. Anand A, Kaur H. Survey on segmentation of Brain Tumor: A review of literature, International Journal of Advanced Research in Computer and Communication Engineering. January 2016; 5(1):79-82. DOI Crosssref.

5. Prastawa M, Bullitt E, Ho S, Gerig G. A brain tumor segmentation framework based on outlier detection, Medical Image Analysis. 2004; 8:275-83. Crossref.

6. Vijayakumar C, Damayanti G, Pant R, Sreedhar CM. Segmentation and grading of brain tumors on apparent diffusion coefficient images using self organizing maps, Computerized Medical Imaging and Graphics. 2007; 31:473-84. Crossref. PMid: 17572068.

7. Corso JJ, Sharon E, Dube S, El-Saden S, Sinha U, Yuille A. Efficient multilevel brain tumor segmentation with integrated bayesian model classification, IEEE Transactions on Medical Imaging. 2008; 27:629-40. Crossref. PMid: 18450536.

8. Zhao L, Jia K. Multiscale CNNs for Brain Tumor segmentation and diagnosis, Computational and Mathematical Methods in Medicine. 2016; 1-7. https://doi. org/10.1155/2016/8356294, Crossref.

9. Amin S, Megeed M. Brain tumor diagnosis systems based on artificial neural networks and segmentation using MRI. 8th International Conference on Informatics and Systems (INFOS); 2012. p. 119-24.

10. Naveen A, Velmurugan T. Identification of calcification in MRI brain images by k-Means Algorithm, Indian Journal of Science and Technology. 2015; 8(29):1-7. Crossref.

11. Lee TS. Image representation using $2 \mathrm{D}$ Gabor wavelets, IEEE Trans. Pattern Analysis and Machine Intelligence. 1996; 18(10):959-71. Crossref.

12. Demirhan A, Toru M, GulerI. Segmentation of tumor and edema along with healthy tissues of brain using wavelets and neural networks, IEEE Journal of biomedical and health informatics. 2015; 19(4):1451-58. Crossref. PMid: 25265636.

13. Alhoniemi E, Himberg J, Parhankangas J, Vesanta J. SOM toolbox for MATLAB. Date accessed: 18/02/2000. Available at: http://www.cis.hut.fi/projects/somtoolbox.

14. Kohonen T. The Self-Organizing Maps, 3rd Ed. Berlin, Germany: Springer-Verlag; 2002. PMCid: PMC125026.

15. Shafarenko L, Petrou M, Kittler J. Automatic watershed segmentation of randomly textured color images, IEEE Transactions on Image Processing. 1997; 6(11):1530-44. Crossref.

16. Serra J. Image Analysis and Mathematical Morphology. Academic Press, New York; 1982. PMCid: PMC1163622. 